\title{
A Stable, Rational $Q R$ Algorithm for the Computation of the Eigenvalues of an Hermitian, Tridiagonal Matrix
}

\author{
By Christian H. Reinsch
}

\begin{abstract}
The most efficient program for finding all the eigenvalues of a symmetric matrix is a combination of the Householder tridiagonalization and the $Q R$ algorithm. The latter, if carried out in a natural way, requires $4 n$ additions, $10 n$ multiplications, $2 n$ divisions, and $n$ square roots per iteration ( $n$ the order of the matrix). In 1963, Ortega and Kaiser showed that the process can be carried out using no square roots (and saving $7 n$ multiplications). However, their algorithm is unstable and several modifications were suggested to increase its accuracy. We, too, want to give such a modification together with some examples demonstrating the achieved accuracy.
\end{abstract}

1. Introduction. In 1961 Francis [4] proposed the $Q R$ transformation, an offspring of Rutishauser's $L R$ transformation [8], for the computation of the eigenvalues of a general matrix. He considered his method to be inefficient for Hermitian matrices but, fortunately, it soon turned out that, contrary to his original opinion, the method is especially efficient for this class of matrices, provided the given matrix is first reduced by Householder's method to real tridiagonal form and provided that shifts are used to accelerate the rate of convergence. (A description of this technique can be found in [10], for tested ALGOL programs see [6], [3], [2], the properties of the now generally adopted shift are described in [11].)

Ortega and Kaiser [7] pointed out that by avoiding square roots the efficiency of this algorithm can be further increased (though if all eigenvalues are to be computed, it is already superior to all other known methods). The algorithm which they proposed, however, was unstable and several modifications were suggested (e.g., [9], [5] and others, not published). We, too, want to give such a modification here, together with some examples demonstrating the achieved accuracy.

2. The Algorithm. Let $A$ be the shifted matrix with diagonal entries $a_{1}, \cdots, a_{n}$ and subdiagonal entries $b_{1}, \cdots, b_{n-1}$. As is well known, a $Q R$ step consists of the orthogonal-triangular decomposition, $A=Q R$, and the recombination in reversed order, $\bar{A}=R Q$. For tridiagonal matrices, the decomposition is usually done by the application of $n-1$ plane rotations $P_{1}^{T}, \cdots, P_{n-1}^{T}$ from the left to $A$ to produce the upper triangular matrix $R$ with diagonal $r_{1}, \cdots, r_{n}$, first superdiagonal $q_{1}, \cdots, q_{n-1}$,

Received November 13, 1970, revised February 1, 1971.

AMS 1970 subject classifications. Primary 65F15.

Key words and phrases. Hermitian matrix, symmetric matrix, tridiagonal matrix, all eigenvalues, $Q R$ transformation. 
and second superdiagonal $t_{1}, \cdots, t_{n-2}$. If

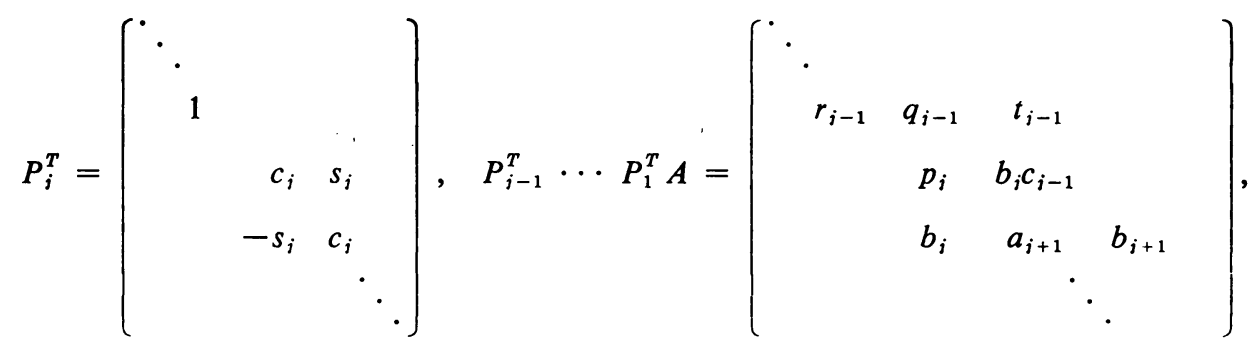

then it follows by induction that

$$
\begin{aligned}
\quad p_{1}=a_{1}, \quad c_{0}=1 ; & \\
r_{i} & =\left(p_{i}^{2}+b_{j}^{2}\right)^{1 / 2}, \\
c_{i} & =p_{i} / r_{i}, \\
s_{i} & =b_{i} / r_{i}, \\
q_{i} & =c_{j} c_{i-1} b_{i}+s_{i} a_{i+1},
\end{aligned}
$$

$$
r_{n}=p_{n}
$$

For the recombination, we have in the tridiagonal case $\bar{A}=R P_{1} \cdots P_{n-1}$ giving

$$
\begin{aligned}
\bar{a}_{i} & =r_{j} c_{i-1} c_{i}+q_{j} s_{i}, \\
& =p_{i} c_{i-1}+q_{i} s_{i}, \quad j=1, \cdots, n-1 ; \\
\bar{b}_{i} & =r_{i+1} s_{i},
\end{aligned}
$$

$$
\bar{a}_{n}=r_{n} c_{n-1} .
$$

Thus, $4 n$ additions, $10 n$ multiplications, $2 n$ divisions, and $n$ square roots are necessary per iteration. This algorithm is realized in the programs of [2]. To avoid square roots, Ortega and Kaiser introduced the quantities

$$
h_{i}=p_{j} c_{i-1}
$$

(which they call $\gamma_{i}$ ) and computed $p_{i}^{2}$ from $h_{i}^{2} /\left(1-s_{i-1}^{2}\right)$ which is obviously inaccurate if $s_{i-1}^{2}$ is near to unity. We prefer to compute a quantity $g_{i}$ defined by

$$
g_{i}=p_{i} / c_{i-1}
$$

and to compute $h_{i}$ from it. From the last equation of (2) and from $c_{i} b_{i}=s_{i} p_{i}$ one obtains the recurrence relation

$$
g_{i+1}=a_{i+1}-s_{j} c_{j-1} b_{i} / c_{i}=a_{i+1}-s_{j} c_{i-1} b_{i}^{2} / s_{j} p_{i}=a_{i+1}-b_{i}^{2} / g_{i},
$$

while according to Ortega and Kaiser, the second equation of (4) can be transformed into

$$
\bar{a}_{i}=h_{j}+c_{j} c_{i-1} b_{j} s_{i}+s_{i} a_{i+1} s_{i}=h_{i}+s_{i}^{2}\left(h_{i}+a_{i+1}\right) .
$$


Thus, the algorithm without square roots becomes

$$
\begin{aligned}
& \quad g_{1}=h_{1}=a_{1}, \quad s_{0}^{2}=0 ; \\
& p_{i}^{2}=g_{i} h_{i}, \\
& r_{i}^{2}=p_{i}^{2}+b_{i}^{2}, j=1, \cdots, n-1 ; \\
& \bar{b}_{i-1}=r_{i}^{2} s_{i-1}^{2} \quad(j \neq 1), \\
& s_{i}^{2}=b_{i}^{2} / r_{i}^{2}, \\
& \bar{a}_{i}=h_{i}+s_{j}^{2}\left(h_{i}+a_{i+1}\right), \\
& g_{i+1}=a_{i+1}-b_{i}^{2} / g_{i}, \\
& h_{i+1}=g_{i+1} p_{i}^{2} / r_{i}^{2}, \\
& \quad a_{n}=h_{n}, \quad \bar{b}_{n-1}^{2}=g_{n} h_{n} s_{n-1}^{2} .
\end{aligned}
$$

Note that the squares of the subdiagonal elements rather than the elements themselves are the given data. This algorithm needs $4 n$ additions, $4 n$ multiplications, and $3 n$ divisions ( $n$ additions less than Ortega and Kaiser but $n$ multiplications and $n$ divisions more). The iteration is repeated until the last off-diagonal entry becomes smaller than a given tolerance $\Delta$.

It should be mentioned that the $g_{i}$ are the well-known quotients of consecutive principal minors of the shifted matrix $A$, which are also used in the bisection process [1]. This can be used to advantage to assign ordinals to computed eigenvalues. As is the case there, a vanishing $g_{i}$ has to be replaced by a small nonzero value $\delta$ equivalent to a perturbation of the diagonal entry $a_{i}$. $\delta$ has to be chosen smaller than $2 \Delta$ in order to avoid indefinite cycling: if $g_{n}$ is replaced by $\delta$ then $\bar{b}_{n-1}^{2}=\delta^{2} s_{n-1}^{2} c_{n-1}^{2} \leqq \delta^{2} / 4<\Delta^{2}$, and the iteration terminates. In any case, the computed values of $g_{j}(j=1, \cdots, n)$ are always the exact values corresponding to slightly modified entries of the matrix $A$. Rounding errors in the evaluation of the remaining expressions are obviously harmless. For technical reasons, a decomposition starting with the lower end of the tridiagonal matrix is preferable (called the " $Q L$ algorithm"). This is merely achieved by the replacement

$$
\begin{gathered}
d_{n}, \cdots, d_{1} \text { for } a_{1}, \cdots, a_{n}, \\
e_{n-1}^{2}, \cdots, e_{1}^{2} \text { for } b_{1}^{2}, \cdots, b_{n-1}^{2},
\end{gathered}
$$

(and similarly for the entries of $\bar{A}$ ). Introducing the fake quantity $e_{n}^{2}$ we obtain the procedure (without shift):

$$
\begin{gathered}
g:=h:=d_{n} ; \quad s^{2}:=0 ; \\
i:=n-1(-1) 1: \\
p^{2}:=g \times h ; \quad r^{2}:=p^{2}+e_{i}^{2} ; \\
\bar{e}_{i+1}^{2}:=s^{2} \times r^{2} ; \quad s^{2}:=e_{i}^{2} / r^{2} ; \\
\bar{d}_{i+1}:=h+s^{2} \times\left(h+d_{i}\right) ; \\
g:=d_{i}-e_{i}^{2} / g ; \quad h:=g \times p^{2} / r^{2} ; \\
\bar{d}_{1}:=h ; \quad \bar{e}_{1}^{2}:=g \times h \times s^{2} .
\end{gathered}
$$


The computation of the shift, the tests for splitting and convergence can be done as in the usual $Q R$ algorithm (see [2]).

3. Test Examples. The algorithm (9) was embedded in the organizational scheme of procedure TQL1 [2]. The following numerical results were obtained on the AEG-TELEFUNKEN computer TR-4 of the Leibnitz-Rechenzentrum der Bayerischen Akademie der Wissenschaften, München, with machine precision $2^{-35} \simeq$ $2.91_{10^{-11}}$. Two consecutive machine numbers in the interval $[1,16)$ have a distance $2^{-34} \simeq 5.82_{10^{-11}}$, and this would be the appropriate unit to measure most of the errors $\tilde{\lambda}_{i}-\lambda_{i}$ listed below. Rounded decimal equivalents are listed in sequence as they were computed.

First Example.

$$
\begin{array}{ll}
\text { order: } & 5, \\
\text { diagonal: } & (0,0,0,0,0), \\
\text { subdiagonal: } & (1,1,1,1) .
\end{array}
$$

This example served as a test for formal correctness. In the first iteration $g_{2}$ and $g_{5}$ become zero for the chosen shift and have to be replaced by a small tolerance quantity.

\begin{tabular}{ccc}
\hline Computed eigenvalues $\tilde{\lambda}_{i}$ & $\tilde{\lambda}_{i}-\lambda_{i}$ & Iterations \\
\hline-0.999999999996 & 0.4 \\
1.0000000000 & 0 \\
$-5.14_{10-12}$ & -0.5 \\
1.7320508077 & 11.2 \\
-1.7320508075 & 6.3 & 1 \\
\end{tabular}

Second Example. Wilkinson's matrix $W_{21}^{-}$,

order: $\quad 21$,

diagonal: $\quad(10,9, \cdots,-9,-10)$,

subdiagonal: $\quad(1, \cdots, 1)$. 
This example was chosen since the exact eigenvalues were known to 24 decimal places.

\begin{tabular}{|c|c|c|}
\hline Computed eigenvalues $\tilde{\lambda}_{i}$ & $\tilde{\lambda}_{i}-\lambda_{i}$ & Iterations \\
\hline 10.746194183 & -0.8 & 3 \\
\hline 9.2106786472 & -11.9 & 2 \\
\hline 8.0389411193 & -3.3 & 2 \\
\hline 7.0039520027 & 1.1 & 2 \\
\hline 6.0002256802 & 5.5 & 2 \\
\hline 5.0000081589 & 17.9 & 2 \\
\hline 4.0000002049 & -12.1 & 2 \\
\hline 3.0000000037 & -8.3 & 2 \\
\hline 2.0000000001 & 0.4 & 2 \\
\hline 0.99999999999 & -1.2 & 2 \\
\hline $4.014_{10^{-11}}$ & $4.0\} \times 10^{-11}$ & 2 \\
\hline-1.0000000005 & -46.5 & 1 \\
\hline-2.0000000001 & -6.2 & 2 \\
\hline-3.0000000040 & -15.0 & 1 \\
\hline-4.0000002057 & -6.3 & 2 \\
\hline-5.0000081591 & -4.1 & 1 \\
\hline-6.0002256806 & -4.6 & 2 \\
\hline-7.0039520030 & $-3.6\} \times 10^{-10}$ & 1 \\
\hline-8.0389411197 & -4.3 & 1 \\
\hline-9.2106786491 & -17.4 & 1 \\
\hline \multirow[t]{2}{*}{-10.746194185} & -20.9 & 0 \\
\hline & & 35 \\
\hline
\end{tabular}

Third Example. Wilkinson's matrix $W_{21}^{+}$,

$$
\begin{array}{ll}
\text { order: } & 21, \\
\text { diagonal: } & (10,9, \ldots, 0, \cdots, 9,10), \\
\text { subdiagonal: } & (1, \cdots, 1) .
\end{array}
$$

Here, too, were the eigenvalues known to 24 decimals. The matrix has a number of close pairs of eigenvalues, and earlier algorithms for the rational $Q R$ transformation gave only poor results. 


\begin{tabular}{|c|c|c|}
\hline Computed eigenvalues $\bar{\lambda}_{1}$ & $\tilde{\lambda}_{i}-\lambda_{i}$ & Iterations \\
\hline 10.746194183 & -0.8 & 3 \\
\hline 10.746194183 & 5.0 & 2 \\
\hline 9.2106786473 & -3.3 & 2 \\
\hline 9.2106786473 & -8.9 & 1 \\
\hline 8.0389411157 & -9.1 & 3 \\
\hline 8.0389411228 & -6.3 & 0 \\
\hline 7.0039517986 & -1.6 & 3 \\
\hline 7.0039522095 & -4.0 & 0 \\
\hline 6.0002175223 & -0.5 & 3 \\
\hline 6.0002340316 & 0.0 & 0 \\
\hline 5.0002444249 & $-9.1\} \times 10^{-11}$ & 3 \\
\hline 4.9997824777 & -6.3 & 1 \\
\hline $4.00435 \quad 40235$ & 10.8 & 3 \\
\hline 3.9960482015 & 13.2 & 1 \\
\hline 3.0430992925 & -8.3 & 3 \\
\hline 2.9610588842 & -2.5 & 1 \\
\hline 2.1302092192 & -18.6 & 2 \\
\hline 1.7893213524 & -31.7 & 1 \\
\hline 0.94753436752 & -0.7 & 2 \\
\hline 0.25380581678 & -31.9 & 1 \\
\hline$-1.12544 \quad 15223$ & -21.8 & 0 \\
\hline
\end{tabular}

Fourth Example.

$$
\begin{array}{ll}
\text { order: } & 21, \\
\text { diagonal: } & (0,0,0,0,5,5, \cdots, 5,5,0,0,0,0), \\
\text { subdiagonal: } & (1, \cdots, 1) .
\end{array}
$$

This matrix has also several close pairs of eigenvalues. The same method in double precision was used to compute them in sufficient accuracy to permit a reliable computation of $\tilde{\lambda}_{i}-\lambda_{i}$. 


\begin{tabular}{|c|c|c|}
\hline Computed eigenvalues $\tilde{\lambda}_{i}$ & $\tilde{\lambda}_{i}-\lambda_{i}$ & Iterations \\
\hline-0.68610208438 & -1.0 & 6 \\
\hline-0.68610208455 & -1.2 & 0 \\
\hline-1.6413343818 & -2.1 & 2 \\
\hline-1.6413343820 & -18.7 & 1 \\
\hline 0.53719550200 & -18.6 & 3 \\
\hline 0.53719549767 & -18.7 & 0 \\
\hline 1.5804833100 & -2.3 & 3 \\
\hline 1.5804833817 & -1.9 & 0 \\
\hline 3.0542418341 & 4.9 & 4 \\
\hline 3.2130677750 & 6.3 & 2 \\
\hline 3.4656821635 & $2.7\} \times 10^{-11}$ & 2 \\
\hline 3.7963179956 & 5.0 & 2 \\
\hline 4.1860204350 & 9.1 & 2 \\
\hline 4.6139505337 & -4.2 & 2 \\
\hline 5.0583784312 & -5.1 & 2 \\
\hline 5.4975503169 & -4.0 & 2 \\
\hline 5.9105127679 & 14.1 & 2 \\
\hline 6.2779084969 & 22.7 & 2 \\
\hline 6.5827245938 & 10.9 & 2 \\
\hline 6.8109624646 & 10.3 & 1 \\
\hline 6.9521974335 & 9.0 & 0 \\
\hline
\end{tabular}

Mathematisches Institut der Technischen Universität München Arcisstrasse 21 8000 München 2, Germany

1. W. Barth, R. S. Martin \& J. H. Wilkinson, "Calculation of the eigenvalues of a symmetric tridiagonal matrix by the method of bisection," Numer. Math., v. 9, 1967, pp. 386-393.

2. H. Bowdler, R. S. Martin, C. Reinsch \& J. H. Wilkinson, "The $Q R$ and $Q L$ algorithms for symmetric matrices," Numer. Math., v. 11, 1968, pp. 293-306.

3. D. J. MUELLER, "Householder's method for complex matrices and eigensystems of hermitian matrices," Numer. Math., v. 8, 1966, pp. 72-92. MR 33 \#872.

4. J. G. F. FranCIS, "The $Q R$ transformation: A unitary analogue to the $L R$ transformation. I, II," Comput. J., v. 4, 1961/62, pp. 265-271, 332-345. MR 23 \#B3143.

5. W. KaHAN, Accurate Eigenvalues of a Symmetric Tri-Diagonal Matrix, Technical Report \#CS41, Computer Science Dept., Stanford University, Stanford, Calif., 1966.

6. R. S. MARTIN, C. REINSCH \& J. H. WilKINSON, "Householder's tridiagonalization of a symmetric matrix," Numer. Math., v. 11, 1968, pp. 181-195.

7. J. M. ORTEGA \& H. F. KAISER, "The $L L^{T}$ and $Q R$ methods for symmetric tridiagonal matrices," Comput. J., v. 6, 1963/64, pp. 99-101. MR 27 \#6379.

8. H. RUTISHAUSER, "Solution of eigenvalue problems with the $L R$-transformation," Nat. Bur. Standards Appl. Math. Ser., v. 49, 1958, pp. 47-81. MR 19,770.

9. H. Rutishauser, "Correspondence to the Editor," Comput. J., v. 6, 1963, p. 133.

10. J. H. WILKInson, The Algebraic Eigenvalue Problem, Clarendon Press, Oxford, 1965. MR 32 \# 1894.

11. J. H. WILKINSON, "Global convergence of tridiagonal $Q R$ algorithm with origin shifts," Linear Algebra and Appl., v. 1, 1968, pp. 409-420. MR 38 \#2938. 